\title{
Long-term outcome after local recurrence of soft tissue sarcoma: a retrospective analysis of factors predictive of survival in 135 patients with locally recurrent soft tissue sarcoma
}

\author{
A Daigeler ${ }^{1}$, I Zmarsly ${ }^{1}$, T Hirsch ${ }^{1}$, O Goertz ${ }^{1}$, H-U Steinau ${ }^{1}$, M Lehnhardt ${ }^{1}$ and K Harati ${ }^{\star}, 1$ \\ ${ }^{1}$ Department of Plastic Surgery, Burn Center, Hand Center, Sarcoma Reference Center, BG-University Hospital Bergmannsheil, \\ Bochum, Germany
}

Background: The aim of this study was to identify prognostic indicators of survival in patients with locally recurrent soft tissue sarcoma (STS) through a long-term follow-up.

Methods: We retrospectively assessed the relationship between post-recurrence survival (PRS) and potential prognostic factors in 135 patients who had experienced local recurrence, which was suitable for further surgical treatment. The median follow-up time after initial recurrence was 12.3 years (95\% confidence interval (Cl): 10.4-14.2 years).

Results: The 5-year estimate of the PRS rate was 53.1\% (95\% Cl: 44.3-61.2\%) for the entire series. Patients with negative margins after the final surgery experienced improved survival compared with patients with positive margins (5-year survival: $46.7 \%$ (35.2-57.5\%) vs 35.5\% (23.4-47.8\%); $P=0.01)$. In a multivariate analysis, the significant prognostic indicators for PRS were histologic grade, tumour site, time to initial recurrence, the number of recurrences and the surgical margin status attained at the last resection.

Conclusions: Complete surgical resection with microscopically clear margins is desirable in patients with locally recurrent STS. However, when achieving clear surgical margins will require major functional impairment of the extremity, a radical surgical approach should be weighed for the patient in each case.

Soft tissue sarcomas (STSs) are a heterogeneous group of rare malignant tumours that account for approximately 1\% of all adult malignancies in Europe and the United States (Weitz et al, 2003; Fernebro et al, 2006; Jemal et al, 2007).

In patients with primary diagnosed STS, the therapy of choice involves limb-sparing surgical resection with clear margins, usually followed by radiation treatment to decrease local recurrence (Singer et al, 2000; Kaushal and Citrin, 2008). There have been several analyses of the prognostic factors influencing overall survival in patients with primary STS (Collin et al, 1987; Gaynor et al, 1992; Pisters et al, 1996; Pisters and Pollock, 1999; Matsumoto et al, 2003). Among these factors, histologic grade, depth, anatomic site, tumour size and histologic subtype are considered the most significant for overall survival. The achievement of negative surgical margins in primary STS has been determined to be an important factor for improving local disease control. However, in the case of local recurrence, the attainment of negative surgical margins may require extensive surgery and could 
result in a loss of extremity function. Although reconstructive plastic surgery can frequently reduce functional impairment and cover soft tissue defects, particularly in cases of large tumour size or location adjacent to critical anatomic structures, the surgical approach to attaining negative margins in locally recurrent disease is associated with considerable morbidity. Furthermore, the impact of negative surgical margins on overall survival in locally recurrent STS is still a subject of debate, and published studies investigating the clinical significance of surgical margins in locally recurrent STS have presented inconsistent results (Sadoski et al, 1993; Karakousis et al, 1996; Ueda et al, 1997; Karakousis and Driscoll, 1999; Trovik et al, 2000a, b, 2001; Stojadinovic et al, 2002a, b; Eilber et al, 2003; Gronchi et al, 2005; Novais et al, 2010). In addition, the reported follow-up data in these studies were not restricted to patients with local recurrence and included the time between primary diagnosis and initial local recurrence, without specifying the post-recurrence follow-up length. However, longer follow-up periods seem reasonable, especially for slow-growing STS subtypes. Therefore, the question remains whether aggressive local disease control would have a positive long-term impact on post-recurrence survival (PRS) in STS patients.

The aim of this study was to identify prognostic indicators of survival in patients with locally recurring STS by reviewing our institutional experience. In particular, we focused on the effect of surgical margins on disease outcome after initial recurrence and definitive surgical treatment.

\section{MATERIALS AND METHODS}

Patients. Between January 1990 and September 2007, 178 adult patients with locally recurrent STS of the extremities or trunk were treated at our institution and recorded in the prospective database of the Soft Tissue Sarcoma Register of the BG University Hospital Bochum. These patients experienced locally recurrent disease at least 3 months after definitive surgery on the primary tumour, which had been performed at our or other institutions. Patients from other institutions were subsequently referred to our centre after the diagnosis of local recurrence. All 178 patients had locally recurrent tumours, which were suitable for further surgical resection. From this entire group, we excluded dermatofibrosarcoma protuberans (6 patients), as this STS subtype has a low metastatic potential. Furthermore, 37 patients, including patients from foreign countries, were lost to follow-up. We restricted analyses to participants with full outcome information. The remaining 135 patients were assessed, and their clinicopathological characteristics are summarised in Tables 1, 2 and 3. Patient followup was obtained from our STS database, medical records and patient correspondence.

All data were obtained from the Soft Tissue Sarcoma Register of the BG University Hospital Bochum, which was initialised in 1991. Patients gave a signed informed consent, which was approved by the ethics committee of the BG University Hospital Bochum.

Treatment. The goal of surgical treatment for all patients was resection of the recurrent tumour with clear margins according to the preoperative imaging results. This resection included the scars from previous surgeries, biopsies and wound drainage. If necessary, local or free flaps were used for coverage of the resulting defects.

Based on prognostic factors predicting an increased risk of disease progression, several patients received after surgical treatment of primary tumour adjuvant radiation and/or chemotherapy using generally anthracycline-based regimens. The indication for adjuvant radiation or chemotherapy was given at the discretion of the interdisciplinary tumour board of either our institution or the referring institutions.
Thirty-six patients with G2 and G3 tumours received adjuvant radiotherapy after resection of their primary tumour with a median overall dose of 60.9 Gy (range: 30.6-70.0). Further 34 patients underwent first adjuvant radiotherapy after initial or second local recurrence with a median overall dose of $62.3 \mathrm{~Gy}$ (range 44.1-68.0). Five patients received adjuvant chemotherapy of primary tumour. Four of them were treated with doxorubicin combined with ifosfamide and one of them received epirubicin with ifosfamide.

Histopathological classification. All tumours were diagnosed and classified using the guidelines of the French Federation of Cancer Centres (FNCLCC) and the latest World Health Organisation (WHO) by the Institute of Pathology at the Ruhr University of Bochum, Germany (Fletcher, 2006). Both the Institute of Pathology and the Department of Plastic Surgery are part of the Sarcoma Reference Center at the Ruhr University of Bochum. All pathology slides were analysed or reviewed for consensus diagnosis by experienced soft tissue pathologists. In specialised cases, an expert second opinion was obtained in Germany (Professor Katenkamp, Jena) or in the United States (Professor Fletcher, Boston). The following histopathological types were included in this study: liposarcoma, pleomorphic sarcoma not otherwise specified (NOS; formerly known as malignant fibrous histiocytoma, MFH), leiomyosarcoma, fibrosarcoma, myxofibrosarcoma, clear cell sarcoma, spindle cell sarcoma, synovial sarcoma and malignant peripheral nerve sheath tumour.

Statistical analysis. All patients were retrospectively analysed regarding possible prognostic factors influencing survival after initial local recurrence (Table 1). PRS was defined as the time period from the date of surgery for initial local recurrence to the date of death from any cause. Survival rates were estimated according to the Kaplan-Meier method with respective 95\% confidence intervals (95\% CIs) and were compared using the logrank test. Multivariate analyses were performed using the Cox proportional hazards model. Variables that were associated with $P<0.1$ in the univariate analysis were included in the multivariate regression to assess independent prognostic factors for survival. $P<0.05$ was considered statistically significant. The data analysis was performed using the statistical program Stata (Version 11.2, StataCorp, College Station, TX, USA).

\section{RESULTS}

Patient characteristics and surgical margins. The mean age at the time of initial recurrence was 58.5 years (range 16.8-87.9). There were 70 men $(51.9 \%)$ and 65 women (48.1\%). Tumours were located in the lower extremities in 78 patients (57.8\%); in the upper extremities in 35 patients (25.9\%); and in the trunk, including the limb girdles, in 22 patients $(16.3 \%)$. The distribution of the histologic grading was G1 in 20 cases (14.8\%), G2 in 55 (40.7\%) and $\mathrm{G} 3$ in $60(44.4 \%)$. In total, 45 patients had one local recurrence, whereas 90 patients had two or more local recurrences (range 1-29). Moreover, 123 patients had only localised disease at the time of initial recurrence, and 12 presented with concurrent metastatic disease. Over time, 42 more patients developed distant metastases, and 44 of the 54 patients with distant metastases had pulmonary metastases. The Kaplan-Meier estimated rate of 5-year distant metastasis-free survival after recurrence was $72.9 \%$ (95\% CI: 63.7-80.1).

In order to determine the impact of surgical resection margins on survival, we analysed the two following variables. In 'status of surgical margins after last resection of locally recurrent tumour' (Table 1, Figure 1F), we assessed survival depending on the resection status (R0 vs R1/R2) that was achieved at the last resection of the locally recurrent tumour. The last resection led to 
Table 1. Results of univariate analyses to determine factors predictive of survival after initial recurrence in 135 soft tissue sarcoma patients

\begin{tabular}{|l|l|l|l|l|}
$\mathbf{N}$ & Estimated 1-year PRS $(95 \% \mathrm{Cl})$ & Estimated 2-year PRS $(95 \% \mathrm{Cl})$ & Estimated 5-year PRS $(95 \% \mathrm{Cl})$ & $\boldsymbol{P}(\text { log-rank })^{\mathrm{a}}$ \\
\hline
\end{tabular}

\section{Age (years)}

$<60$

$\geqslant 60$

$85.3(75.1-91.6)$

$91.7(81.1-96.4)$

$66.7(54.8-76.1)$

$81.7(69.3-89.4)$

$53.3(41.4-63.8)$

$54.4(41.0-66.1)$

Sex

Male

Female

$85.7(75.1-92.0)$

$90.8(80.6-95.7)$

$75.7(63.8-84.1)$

$70.8(58.1-80.2)$

$53.8(41.4-64.7)$

$53.8(41.0-65.0)$

Site

Trunk

Extremity

$81.8(58.5-92.8)$

$89.4(82.1-93.8)$

$54.5(32.1-72.4)$

$77.0(68.1-83.7)$

$45.5(24.4-64.3)$

$55.5(45.8-64.1)$

Time from diagnosis to initial recurrence

\begin{tabular}{|c|c|c|}
\hline$<2$ years & 51 & \\
\hline$\geqslant 2$ years & 84 & \\
\hline \multicolumn{3}{|c|}{ Number of local recurrences } \\
\hline
\end{tabular}

\begin{tabular}{l|l|l}
\hline$\leqslant 2$ & 81 & $82.7(72.6-89.4)$
\end{tabular}

\begin{tabular}{l|l|l}
$>2$ & 54 & $96.3(86.0-99.1)$
\end{tabular}

$81.0(70.8-87.9)$

$100(-)$

$63.1(51.8-72.4)$

$90.1(77.9-95.8)$

$45.2(34.4-55.5)$

$68.1(53.3-79.1)$

0.100

\section{Stage at initial recurrence}

\begin{tabular}{|r|r|r|}
\hline Only localised & 123 & 91.1 (84.4-94.9)
\end{tabular}

$58.3(27.0-80.1)$

$76.4(67.9-83.0)$

$41.7(15.2-66.5)$

$47.8(36.6-58.2)$

$62.9(48.6-74.2)$

$64.2(52.7-73.5)$

$87.0(74.7-93.6)$

0.074

Metastatic

12

tumour

Adju

\begin{tabular}{|l|l|l} 
Yes & 36 & $88.9(73.1-95.7)$ \\
\hline
\end{tabular}

No

$87.9(79.6-92.9)$

$69.4(51.7-81.8)$

$74.7(65.0-82.2)$

$41.7(25.6-57.0)$

$58.4(48.0-67.4)$

Adjuvant chemotherapy of primary tumour

\begin{tabular}{l|r|r} 
Yes & 5 & $60.0(12.6-88.2)$
\end{tabular}

\begin{tabular}{l|l|l} 
No & 130 & $89.2(82.5-93.5)$
\end{tabular}

$60.0(12.6-88.2)$

$73.8(65.4-80.5)$

$40.0(5.2-75.3)$

$54.4(45.4-62.5)$

0.087

Status of surgical margins after last resection of locally recurrent tumour

\begin{tabular}{|l|l|l|}
\hline Negative (R0) & 76 & 90.8 (81.6-95.5) \\
Positive (R1/R2) & 59 & 84.7 (72.7-91.8)
\end{tabular}

$76.3(65.0-84.3)$

$69.5(56.0-79.6)$

$56.2(44.3-66.5)$

$50.8(37.5-62.7)$

Attainment of negative margins (RO) during course of disease

\begin{tabular}{|l|l|l|l|l|l|}
\hline Yes & 94 & $87.2(78.6-92.5)$ & $72.3(62.1-80.2)$ & 0.200 & $52.9(42.3-62.4)$ \\
No & 41 & $90.2(76.1-96.2)$ & $75.6(59.4-86.1)$ & $56.1(39.7-69.6)$ \\
\hline
\end{tabular}

Abbreviations: $\mathrm{Cl}=$ confidence interval; PRS = post-recurrence survival.

${ }^{\text {a } L o g-r a n k ~ t e s t ~ f o r ~ e q u a l i t y ~ o f ~ s u r v i v o r ~ f u n c t i o n s . ~}$

microscopically negative margins (R0) in 76 patients (56.3\%), whereas 59 patients $(43.7 \%)$ were left with positive margins (R1/R2) at the end of surgical therapy. In those cases of positive margins, recurring tumours infiltrated critical anatomic structures, such as large-extremity nerves, or were too advanced and widespread for complete resection.

In 'attainment of negative margins (R0) during course of disease' (Table 1, Figure 1E), we compared the survival of patients in whom R0 status was neither attained in the resection of the primary tumour nor in the following resection(s) of the locally recurrent tumour(s) with the survival of patients who underwent at least one $\mathrm{R} 0$ resection during the course of disease. Here, 41 patients $(30.3 \%)$ underwent only incomplete resection (R1/R2) of local disease since primary diagnosis and therefore lived throughout the course of the disease with residual tumour tissue. Ninetyfour patients (69.6\%) underwent at least one R0 resection since primary diagnosis.
Follow-up. As of February 2013 (cutoff date), the reverse Kaplan-Meier estimate of median follow-up after initial recurrence was 12.3 years (95\% CI: 10.4-14.2; Schemper and Smith, 1996), whereas the minimum post-recurrence follow-up duration was 5 years for patients who were alive at the cutoff date.

Recurrence-free time from primary diagnosis to initial recurrence. The median recurrence-free time calculated from primary diagnosis to initial recurrence was 15.7 months (95\% CI: 10.9-20.0) for the entire cohort (Table 3). Leiomyosarcoma patients had a lower median recurrence-free interval (9.0 months; 95\% CI: 3.8-9.9).

Survival. The total median survival times were 6.2 years after initial recurrence (95\% CI: 4.4-7.6) and 1.5 years after the diagnosis of distant metastasis (95\% CI: 0.93-2.17; Table 3). In the entire series, the 5-year estimate of the PRS rate was 53.1\% (95\% CI: 44.3-61.2), whereas the estimated 5-year rate of survival after 


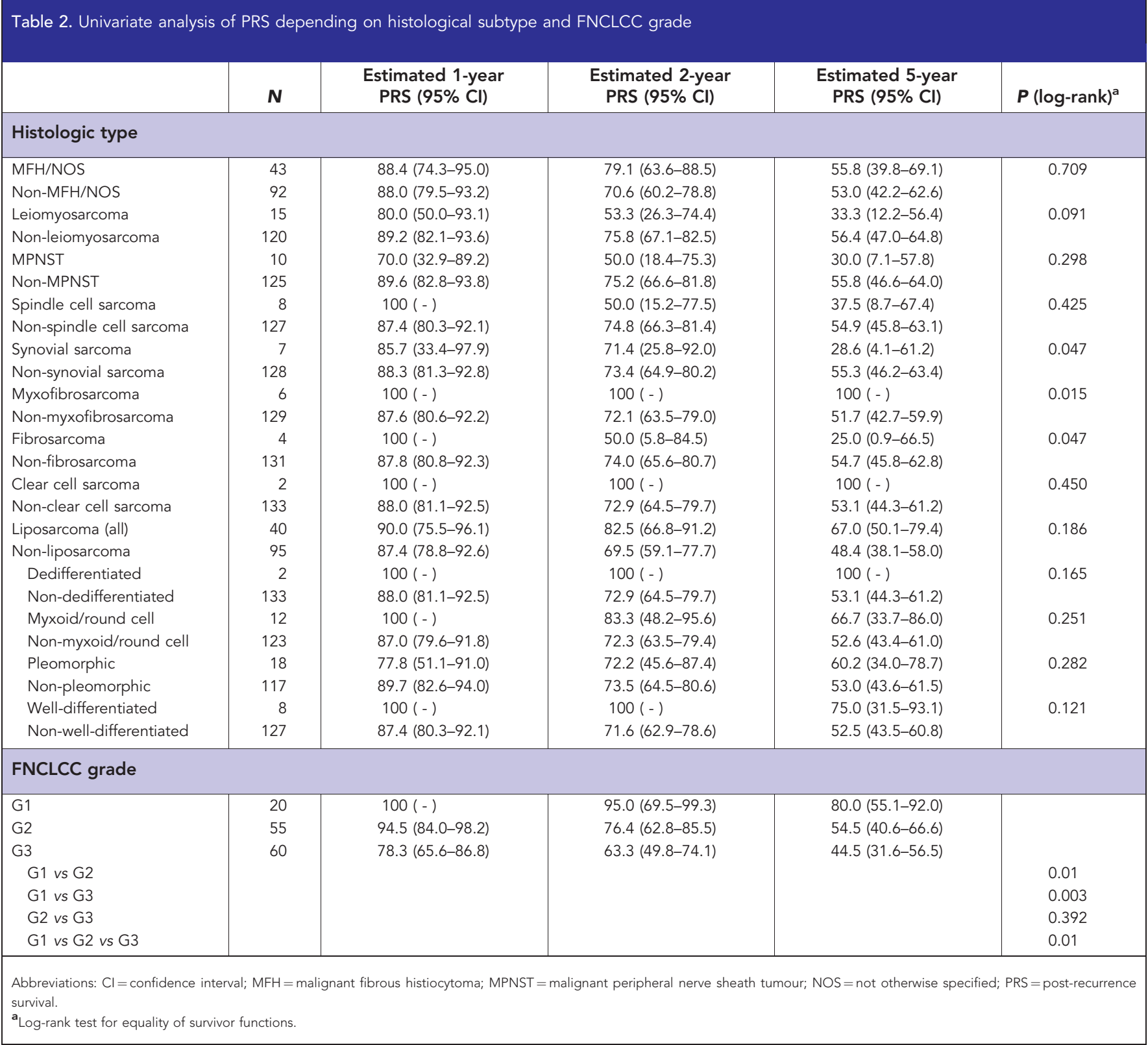

primary diagnosis was $64.7 \%$ (95\% CI: 55.9-72.1). Patients who developed distant metastases had a 5-year survival of $17.0 \%(95 \%$ CI: 8.4-28.2) after the diagnosis of initial metastasis.

Univariate analysis of survival. Regarding the two largest histologic subsets, liposarcoma and NOS (pleomorphic sarcoma NOS; formerly known as MFH) patients had comparable PRS. Similar to findings for other malignancies, patients with low-grade tumours (G1) had more favourable prognoses than did patients with intermediate (G2) or high-grade (G3) lesions. The 5-year PRS rates were estimated to be $80.0 \%$ (95\% CI: 55.1-92.0) for G1 and 44.5\% (95\% CI: 31.6-56.5) for G3 $(P=0.003$; Table 2$)$.

Patients with longer time intervals from primary diagnosis to local recurrence had improved survival (Figure 1C). Initial recurrence, which occurred $\geqslant 2$ years from primary diagnosis, was found to be a predictor of better outcome (5-year PRS of 68.1\%; 95\% CI: 53.3-79.1) compared with earlier recurrence (5-year PRS of $45.2 \%$; 95\% CI: 34.4-55.5; $P=0.042$ ). Notably, patients with more than two resected local recurrences tended to have prolonged PRS compared with patients who developed only one or two recurrences (5-year PRS: 62.9\% (48.6-74.2) vs $47.8 \%$
(36.6-58.2)) (Figure 1D), but the difference was not statistically significant in the univariate analysis $(P=0.074)$. Concurrent metastasis at the time of initial recurrence was associated with significantly worse PRS compared with only localised disease (5-year PRS: 16.7\% (2.7-41.3) vs 57.5\% (39.7-69.6); $P=0.0001$ ). STS arising in the trunk appeared to have a worse prognosis compared with extremity lesions (5-year PRS: 45.5\% (24.4-64.3) vs 55.5\% (45.8-64.1); $P=0.049)$.

Patients who underwent only incomplete resection of local disease since primary diagnosis and therefore lived throughout the course of the disease with residual tumour tissue had an outcome similar to patients who underwent at least one complete resection with the attainment of negative margins (5-year PRS: $56.1 \%$ (39.7-69.6) vs 52.9\% (42.3-62.4); $P=0.2$; Figure 1E). However, the surgical margin status attained by definitive resection of the recurrent tumour influenced PRS and survival after the final surgical treatment (Figure 1F, Figure 2, Table 4); microscopically negative margin status was associated with better PRS compared with positive margin status (5-year PRS: 56.2\% (44.3-66.5) vs $50.8 \%(37.5-62.7))$, although this survival distribution failed to reach statistical significance in the univariate analysis, and a 


\begin{tabular}{|c|c|c|c|c|c|}
\hline & $\mathbf{N}$ & $\begin{array}{l}\text { Person-time in } \\
\text { years after } \\
\text { initial } \\
\text { recurrence }\end{array}$ & $\begin{array}{c}\text { Number } \\
\text { of } \\
\text { deaths }\end{array}$ & $\begin{array}{l}\text { Median recurrence-free time from } \\
\text { primary diagnosis to initial } \\
\text { recurrence in months }(95 \% \mathrm{Cl})\end{array}$ & $\begin{array}{c}\text { Median survival time after } \\
\text { initial recurrence in } \\
\text { years }(95 \% \mathrm{Cl})\end{array}$ \\
\hline $\mathrm{MFH} / \mathrm{NOS}$ & 43 & 315 & 28 & $15.7(6.8-26.0)$ & $6.3(3.9-15.2)$ \\
\hline Leiomyosarcoma & 15 & 57 & 11 & $9.0(3.8-9.9)$ & $2.6(0.9-7.6)$ \\
\hline MPNST & 10 & 54 & 7 & $5.0(1.6-16.9)$ & $2.0\left(0.1-^{a}\right)$ \\
\hline Spindle cell sarcoma & 8 & 49 & 6 & $8.3(2.7-34.8)$ & $1.6\left(1.1-^{\mathrm{a}}\right)$ \\
\hline Synovial sarcoma & 7 & 27 & 7 & $62.6(3.7-108.1)$ & $4.4(0.5-5.4)$ \\
\hline Myxofibrosarcoma & 6 & 56 & 0 & $18.0\left(6.8^{-a}\right)$ & - \\
\hline Fibrosarcoma & 4 & 12 & 4 & $11.0\left(9.5^{-a}\right)$ & $1.3\left(1.3-^{\mathrm{a}}\right)$ \\
\hline Clear-cell sarcoma & 2 & 26 & 1 & $65.0\left(65.0-^{a}\right)$ & $10.9\left(10.9-^{\mathrm{a}}\right)$ \\
\hline Liposarcomas (all) & 40 & 316 & 23 & $21.5(12.3-33.0)$ & $7.6\left(5.2-^{a}\right)$ \\
\hline Dedifferentiated & 2 & 24 & 0 & $4.8\left(4.8-^{\mathrm{a}}\right)$ & - \\
\hline Myxoid/round cell & 12 & 103 & 6 & $12.3(6.6-43.4)$ & $8.5\left(1.7-^{\mathrm{a}}\right)$ \\
\hline Pleomorphic & 18 & 112 & 14 & $16.8(4.7-37.5)$ & $5.6(1.8-7.2)$ \\
\hline Well-differentiated & 8 & 77 & 3 & $54.0(9.4-95.3)$ & - \\
\hline Total & 135 & 912 & 87 & $15.7(10.9-20.0)$ & $6.2(4.4-7.6)$ \\
\hline
\end{tabular}

borderline $P$-value was attained $(P=0.059)$. Considering survival after the last resection, complete resection of the locally recurrent tumour with negative margins resulted in significantly more favourable survival for the entire group (5-year survival after last resection: $46.7 \% \quad(35.2-57.5)$ vs $35.5 \% \quad(23.4-47.8) ; \quad P=0.01$; Figure 2A, Table 4). Only the cohort of patients with concurrent distant metastasis at the point of the last resection did not gain a survival benefit from $\mathrm{R} 0$ resection of the locally recurring tumour.

Multivariate analysis of survival. In the Cox model, significant prognostic factors for PRS were histologic grade, tumour site, the time to initial recurrence, the number of recurrences and the surgical margin status attained at the last resection (Table 5). The hazard ratio (HR) for death after recurrence was 1.8 (95\% CI: 1.02-3.18) for STS arising in the trunk compared with extremity lesions $(P=0.043)$. Recurrence $<2$ years from primary diagnosis was also associated with an increased HR for death after recurrence (HR 1.75; 95\% CI: $1.08-2.81 ; P=0.022$ ). Survival after three or more recurrences was a predictor of a diminished HR (HR 0.48 ; 95\% CI: $0.27-0.84 ; P=0.010)$. Concurrent metastasis at initial recurrence was an indicator of worse survival in the univariate analysis, but this finding was not significant in the multivariate analysis $(P=0.068)$. Patients with $\mathrm{G} 1$ tumours had more favourable survival than patients with G2 or G3 lesions. The Cox model demonstrated that surgical attainment of negative margins at the last resection was associated with a significantly lower HR for death. The HR for negative compared with positive margin status after the last resection was 0.57 (95\% CI: 0.34-0.94; $P=0.026)$.

\section{DISCUSSION}

In more frequent malignancies, such as breast cancer, melanoma or colon carcinoma, large clinical investigations with emphasis on PRS have already been performed. Prognostic determinants of PRS were characterised and based on follow-up data referring to the time after initial recurrence. Furthermore, patients with local recurrence were analysed separately and not mixed with those patients who presented with primary tumours. However, several studies on large series of STS patients have extensively outlined the survival of the subset of patients with recurrent disease. In these studies, the total follow-up after primary diagnosis ranged from 48 to 107 months, including patients without recurrence (Ueda et al, 1997; Stojadinovic et al, 2002a, b; Gronchi et al, 2005). Another large study assessed survival data from 107 patients with locally recurring STS but reported explicit follow-up data only for those patients without recurrence (Trovik et al, 2001). However, an appropriate analysis of the influence of surgical margins on survival in STS patients requires a long-term follow-up after surgical treatment. In a series from Memorial Sloan-Kettering Cancer Center, Lewis et al (1999) revealed that patients with microscopically positive margins after the resection of primary tumours were at higher risk of post-5-year disease-specific mortality. Stojadinovic et al (2002b) and Gronchi et al (2005) made similar observations for patients with positive margins in a large series, indicating that surgical margin status may have a greater prognostic influence later in the natural history. Therefore, the long-term follow-up of patients with positive resection margins is critical in interpreting survival.

Although local recurrence alone is a poor prognostic sign, with reduced survival rates (Pisters et al, 1996; Lewis et al, 1997), numerous clinical and pathologic variables continue to be significant in predicting survival following initial local recurrence. Here, similar to findings observed in other malignancies, late recurrence and low histologic grade were associated with improved survival. Furthermore, STS recurring in extremity locations appeared to have a significantly better prognosis compared with non-extremity lesions in our series. This observation is consistent with previous findings for primary STS. Gutierrez et al (2007) reported that patients with primary extremity lesions had a higher median survival time than those patients with truncal lesions. It seems reasonable that tumours with such different sites may exhibit different clinical behaviour and altered survival, but it should be noted that complete resection of extensive tumours and adequate local control are more difficult to achieve in the trunk wall (Salas et al, 2009). 
A

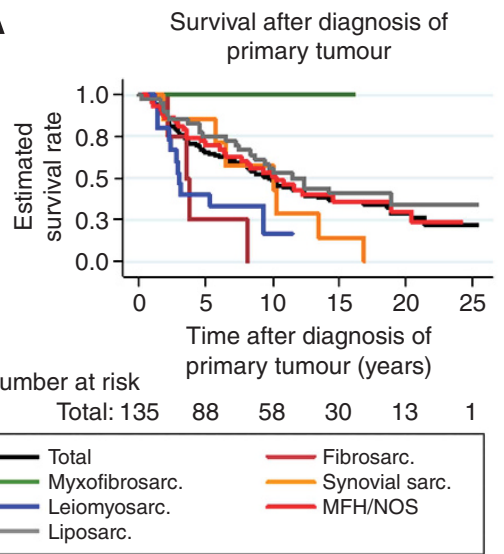

C

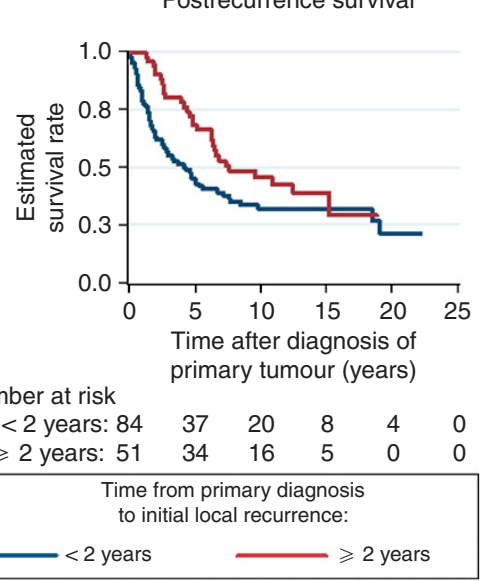

E

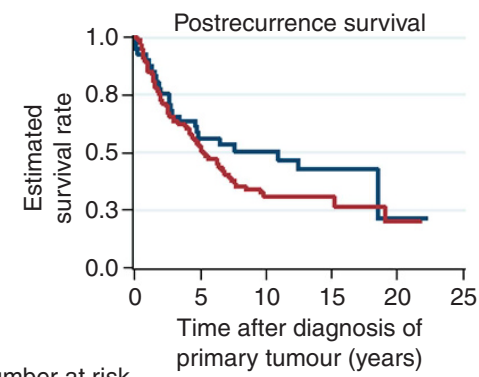

Number at risk

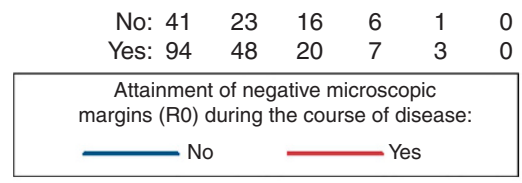

B

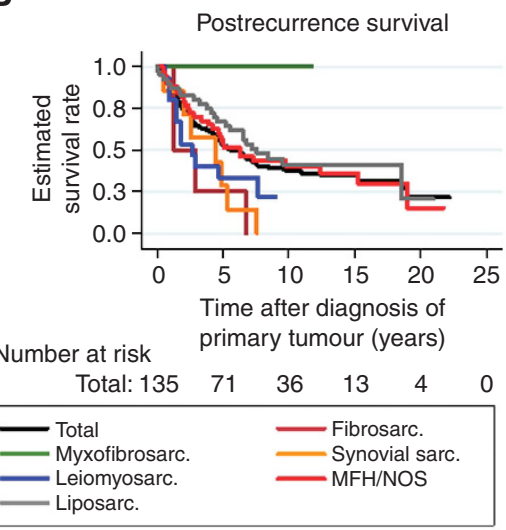

D

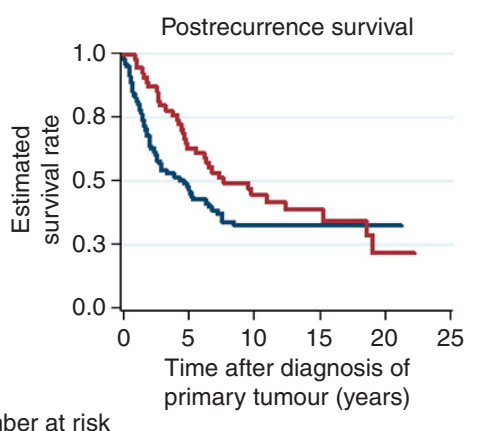

Number at risk

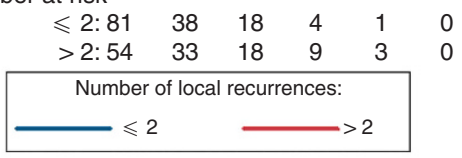

$\mathbf{F}$

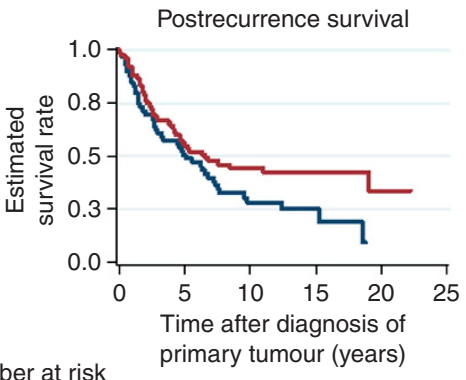

Number at risk

Positive: $\begin{array}{llllll}59 & 29 & 12 & 4 & 0 & 0\end{array}$

Negative: $\begin{array}{llllll}76 & 42 & 24 & 9 & 4 & 0\end{array}$

Status of surgical margins after last resection:

Positive margins (R1/R2)

Negative margins (R0)

Figure 1. Estimated survival curves after primary diagnosis $(\mathbf{A})$ and initial recurrence $(\mathbf{B}-\mathbf{F})$ according to the mentioned factors.

Initially, it may seem surprising that patients who experienced more than three recurrences presented a significantly better outcome than patients with fewer recurrences. This phenomenon can be traced back to the fact that recurrence could only occur successively in those patients with a priori prolonged survival times. Hence, the favourable effect of three or more recurrences on PRS is presumably due to the selection of a patient population that can undergo resection and survive recurrences several times. However, differences in tumour biology can also provide a potential explanation for this observation: tumours that recurred three or more times might have been locally aggressive but failed to facilitate metastasis at an early stage, so that several recurrences could occur in an apparent prolonged survival time. In contrast, biologically more aggressive tumours with higher metastatic potential led to metastatic disease before three or more recurrences could occur resulting in an impaired survival and, thus, fewer survived local recurrences.

Another interesting observation was that only 36 of 115 patients with G2 and G3 tumours received adjuvant radiotherapy after resection of their primary tumour. Retrospectively, we could not determine why adjuvant radiotherapy was not performed in these patients. However, it must be stated that we analysed a selected group of patients where local recurrence definitely occurred. This implies a selection bias that might explain the observation why so many patients did not have adjuvant radiotherapy of their primary tumour and that this lack of adjuvant radiotherapy could be one 
Survival after last resection

Kaplan-Meier estimates

A

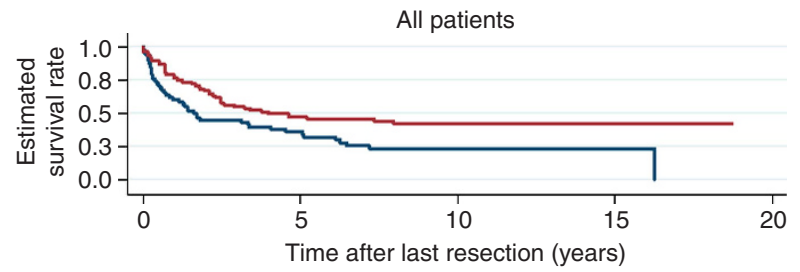

Number at risk

Positive: 59

Negative: 76

18

35

Patients with only localized disease

B

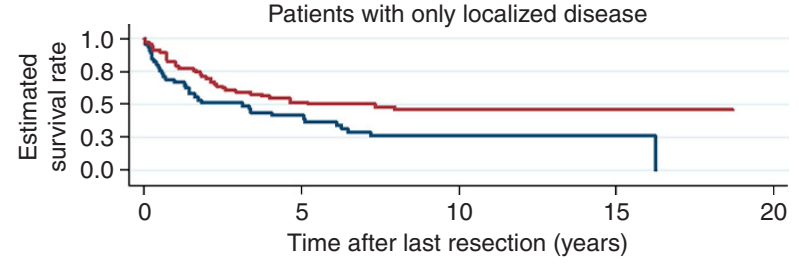

Number at risk

Positive: 46

Negative: 67
17

34

Patients with metastatic disease

C

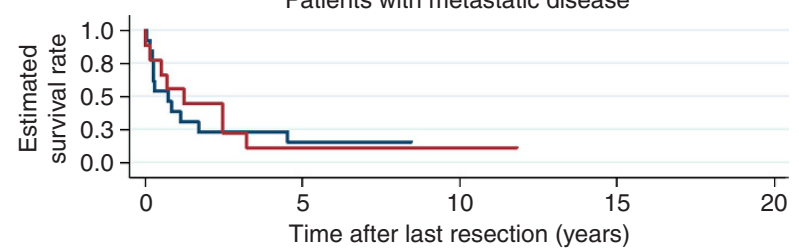

Number at risk

Positive: 13

Negative: 9

\begin{tabular}{l} 
Status of surgical margins after last resection: \\
Positive (R1/R2) Negative (R0) \\
\hline
\end{tabular}

Figure 2. Effects of definitive surgical margins on survival after the last resection in: the overall patient population $(\mathbf{A})$, patients with only localised disease $(\mathbf{B})$ and patients with metastases $(\mathbf{C})$ at the time of the last resection.

potential risk factor for developing local recurrence. The fact that so many patients of this series (79 out of 115) did not receive adjuvant radiotherapy of their primary tumour could be the reason itself why these patients had suffered local recurrence. However, this is only a presumption that cannot be proven by this retrospective analysis.

It is well known that the rarity and heterogeneity of STSs pose diagnostic and therapeutic challenges. Over 50 different histologic subtypes comprise STS, and their impact on patient outcome is equally heterogeneous (Poremba, 2006). In the current series, we sought to determine whether any of the nine encompassed histotypes demonstrated prognostic significance. Here, although certain histotypes were statistically significant predictors of PRS, these findings have to be interpreted with caution because of the small number of patients affected by these histotypes.

One of the main aims in this study was to assess whether complete resection with negative margins has an influence on the long-term prognosis of patients with already recurrent STS. In this series, the surgical margin status that was attained by the final surgery of the local recurrent tumour had prognostic significance for long-term survival. Similar findings were made by Gronchi et al (2005) in a subset of 269 patients with locally recurrent STS through a follow-up of 107 months after primary diagnosis.
In their series, the effect of surgical margins on survival was found to be more striking in patients with already recurrent STS than in patients with primary STS.

These findings might initially suggest an aggressive surgical approach with the goal of attaining negative margins to improve survival. However, it is also probable that recurrent tumours that cannot be completely resected have more aggressive biological features than completely resectable tumours and thus impair the outcome more substantially. In accordance, most of the large studies that analysed prognostic factors in primary STS found a correlation between resection margins and final outcome, but they concluded that it is the inherent aggressiveness of the tumour that dictates both the surgically attainable margin status and the final outcome (Pisters et al, 1996; Trovik et al, 2000a; Stojadinovic et al, 2002a, b; Zagars et al, 2003). They argued that positive margin status is rather a result than a cause of biological aggressiveness and that it does not influence survival directly.

In our study, positive margins per se did not influence survival: in 'attainment of negative margins (R0) during course of disease', we compared the survival of patients in whom R0 status was neither attained in the resection of the primary tumour nor in the following resection(s) of the locally recurrent tumour(s) with the survival of patients who underwent at least one R0 resection during the course of disease. Interestingly, patients who underwent only incomplete resection of local disease since primary diagnosis and therefore lived throughout the course of the disease with residual tumour tissue had similar PRS to patients who underwent at least one complete resection with negative margins. Thus, not every non-R0-resectable, recurrent tumour or positive margin respectively was strictly associated with impaired survival.

In contrast, tumours that had recurred despite previous R0 resection and had not been approachable for R0 resection anymore seemed to progress more aggressively. These recurrent tumours derived from micrometastases in the tissue around the original location, which were left despite previous R0 resection and might be selected out from biologically more aggressive tumour cells, which display higher invasive and metastatic potential and, thus, impeding a R0 resection afterwards and facilitating metastatic disease. However, the impact of viable but primarily histological inapparent residual disease in the post-resection tumour bed needs to be prospectively assessed by determining the influence of the surgically achieved width of the surrounding healthy tissue on recurrence and survival.

Finally, the reservation must be made that our series included only patients with locally recurrent STS, which were suitable for further surgical treatment. Patients with local recurring tumours that could not be approached surgically because of rapid disease progression and therefore with less favourable outcome were not assessed in this study. Consequently, our findings on survival indicators are only applicable to the selected group of patients where further surgical treatment is possible and not to all patients who develop local recurrence after surgical resection. This implies a study selection bias that has to be acknowledged.

In conclusion, this study provides long-term follow-up data that may help clinicians estimate the prognosis of patients experiencing an initial recurrence more accurately and to guide clinical decisions after this emotionally devastating diagnosis. Adverse prognostic features include early recurrence, truncal location and high-grade histology. Furthermore, the data from this study underscore the long-term benefit of negative margins achieved at the end of surgical treatment in patients with locally recurrent STS without distant metastases. The biology of the recurring tumour might dictate the outcome, but given the diminished outcome of patients left with positive margins, surgical efforts should aim to achieve microscopically clear margins during the resection of recurrent tumours. However, we cannot retrospectively conclude that the achievement of negative margins at any cost would have 
Table 4. Univariate analysis of survival after last resection depending on obtained surgical margins at last resection of local recurrent tumour

\begin{tabular}{|c|c|c|c|c|c|}
\hline & $\mathbf{N}$ & $\begin{array}{l}\text { Estimated 1-year survival after } \\
\text { last resection }(95 \% \mathrm{Cl})\end{array}$ & $\begin{array}{l}\text { Estimated 2-year survival } \\
\text { after last resection }(95 \% \mathrm{Cl})\end{array}$ & $\begin{array}{l}\text { Estimated } 5 \text {-year survival } \\
\text { after last resection }(95 \% \mathrm{Cl})\end{array}$ & $P(\log -\text { rank })^{a}$ \\
\hline \multicolumn{6}{|c|}{ Without concurrent distant metastasis at last resection } \\
\hline $\begin{array}{l}\text { Negative margins (R0) } \\
\text { Positive margins (R1/R2) }\end{array}$ & $\begin{array}{l}67 \\
46\end{array}$ & $\begin{array}{l}80.4(68.7-88.1) \\
66.7(50.9-78.4)\end{array}$ & $\begin{array}{l}69.8(57.2-79.4) \\
51.1(35.8-64.5)\end{array}$ & $\begin{array}{l}51.6(39.0-62.8) \\
41.4(26.8-55.4)\end{array}$ & 0.017 \\
\hline \multicolumn{6}{|c|}{ With concurrent distant metastasis at last resection } \\
\hline $\begin{array}{l}\text { Negative margins (R0) } \\
\text { Positive margins (R1/R2) }\end{array}$ & $\begin{array}{r}9 \\
13\end{array}$ & $\begin{array}{l}55.6(20.4-80.5) \\
38.5(14.1-62.8)\end{array}$ & $\begin{array}{l}44.4(13.6-71.9) \\
23.1(5.6-47.5)\end{array}$ & $\begin{array}{l}11.1(0.6-38.8) \\
15.4(2.5-38.8)\end{array}$ & 0.862 \\
\hline \multicolumn{6}{|l|}{ Total } \\
\hline $\begin{array}{l}\text { Negative margins (R0) } \\
\text { Positive margins (R1/R2) }\end{array}$ & $\begin{array}{l}76 \\
59\end{array}$ & $\begin{array}{l}77.5(66.3-85.3) \\
60.3(46.6-71.6)\end{array}$ & $\begin{array}{l}66.8(54.9-76.2) \\
44.8(31.8-57.0) \\
\end{array}$ & $\begin{array}{l}46.7(35.2-57.5) \\
35.5(23.4-47.8)\end{array}$ & 0.010 \\
\hline
\end{tabular}

Table 5. Results of multivariate analysis on post-recurrence survival according to Cox proportional hazard model

\begin{tabular}{|c|c|c|c|}
\hline Category (reference) & Hazard ratio & $95 \% \mathrm{Cl}$ & $\boldsymbol{P}$ \\
\hline Age (years): $\geqslant 60$ (vs $<60)$ & 0.66 & $0.41-1.06$ & 0.087 \\
\hline Time from diagnosis to initial recurrence: $<2$ years ( $v s \geqslant 2$ years) & 1.75 & $1.08-2.81$ & 0.022 \\
\hline Number of recurrence: $>2$ (vs $\leqslant 2$ ) & 0.48 & $0.27-0.84$ & 0.010 \\
\hline Primary chemotherapy: yes (vs no) & 1.98 & $0.86-4.57$ & 0.108 \\
\hline Site: trunk (vs extremity) & 1.80 & $1.02-3.18$ & 0.043 \\
\hline Stage at initial recurrence: localised (vs metastatic) & 0.43 & $0.17-1.07$ & 0.068 \\
\hline Surgical margins after last resection: negative (vs positive) & 0.57 & $0.34-0.94$ & 0.026 \\
\hline \multicolumn{4}{|l|}{ FNCLCC grade } \\
\hline G2 (vs G1) & 2.80 & $1.23-6.40$ & 0.014 \\
\hline G3 (vs G1) & 3.69 & $1.61-8.45$ & 0.002 \\
\hline
\end{tabular}

improved PRS in patients with positive margins. When the goal of achieving clear surgical margins will require amputation or major functional impairment of the extremity, a decision should ultimately be made in each case based on the biology of the STS, the health status of the patient and, last but not the least, the decision of the informed patient.

\section{CONFLICT OF INTEREST}

The authors declare no conflict of interest.

\section{REFERENCES}

Collin C, Godbold J, Hajdu S, Brennan M (1987) Localized extremity soft tissue sarcoma: an analysis of factors affecting survival. J Clin Oncol 5(4): 601-612.

Eilber FC, Rosen G, Nelson SD, Selch M, Dorey F, Eckardt J, Eilber FR (2003) High-grade extremity soft tissue sarcomas: factors predictive of local recurrence and its effect on morbidity and mortality. Ann Surg 237(2): $218-226$.

Fernebro J, Bladstrom A, Rydholm A, Gustafson P, Olsson H, Engellau J, Nilbert M (2006) Increased risk of malignancies in a population- based study of 818 soft-tissue sarcoma patients. Br J Cancer 95(8): 986-990.

Fletcher CD (2006) The evolving classification of soft tissue tumours: an update based on the new WHO classification. Histopathology 48(1): 3-12.

Gaynor JJ, Tan CC, Casper ES, Collin CF, Friedrich C, Shiu M, Hajdu SI, Brennan MF (1992) Refinement of clinicopathologic staging for localized soft tissue sarcoma of the extremity: a study of 423 adults. J Clin Oncol 10(8): 1317-1329.

Gronchi A, Casali PG, Mariani L, Miceli R, Fiore M, Lo Vullo S, Bertulli R, Collini P, Lozza L, Olmi P, Rosai J (2005) Status of surgical margins and prognosis in adult soft tissue sarcomas of the extremities: a series of patients treated at a single institution. J Clin Oncol 23(1): 96-104.

Gutierrez JC, Perez EA, Franceschi D, Moffat Jr FL, Livingstone AS, Koniaris LG (2007) Outcomes for soft-tissue sarcoma in 8249 cases from a large state cancer registry. J Surg Res 141(1): 105-114.

Jemal A, Siegel R, Ward E, Murray T, Xu J, Thun MJ (2007) Cancer statistics, 2007. CA Cancer J Clin 57(1): 43-66.

Karakousis CP, Driscoll DL (1999) Treatment and local control of primary extremity soft tissue sarcomas. J Surg Oncol 71(3): 155-161.

Karakousis CP, Proimakis C, Rao U, Velez AF, Driscoll DL (1996) Local recurrence and survival in soft-tissue sarcomas. Ann Surg Oncol 3(3): 255-260.

Kaushal A, Citrin D (2008) The role of radiation therapy in the management of sarcomas. Surg Clin North Am 88(3): 629-646, viii.

Lewis JJ, Leung D, Casper ES, Woodruff J, Hajdu SI, Brennan MF (1999) Multifactorial analysis of long-term follow-up (more than 5 years) of primary extremity sarcoma. Arch Surg 134(2): 190-194. 
Lewis JJ, Leung D, Heslin M, Woodruff JM, Brennan MF (1997) Association of local recurrence with subsequent survival in extremity soft tissue sarcoma. J Clin Oncol 15(2): 646-652.

Matsumoto S, Ahmed AR, Kawaguchi N, Manabe J, Matsushita Y (2003) Results of surgery for malignant fibrous histiocytomas of soft tissue. Int J Clin Oncol 8(2): 104-109.

Novais EN, Demiralp B, Alderete J, Larson MC, Rose PS, Sim FH (2010) Do surgical margin and local recurrence influence survival in soft tissue sarcomas? Clin Orthop Relat Res 468(11): 3003-3011.

Pisters PW, Leung DH, Woodruff J, Shi W, Brennan MF (1996) Analysis of prognostic factors in 1,041 patients with localized soft tissue sarcomas of the extremities. J Clin Oncol 14(5): 1679-1689.

Pisters PW, Pollock RE (1999) Staging and prognostic factors in soft tissue sarcoma. Semin Radiat Oncol 9(4): 307-314.

Poremba C (2006) [Soft tissue sarcomas: the role of histology and molecular pathology for differential diagnosis]. Verh Dtsch Ges Pathol 90: 59-72.

Sadoski C, Suit HD, Rosenberg A, Mankin H, Efird J (1993) Preoperative radiation, surgical margins, and local control of extremity sarcomas of soft tissues. J Surg Oncol 52(4): 223-230.

Salas S, Bui B, Stoeckle E, Terrier P, Ranchere-Vince D, Collin F, Leroux A, Guillou L, Michels JJ, Trassard M, Valo I, Robin YM, Marques B, Brouste V, Coindre JM (2009) Soft tissue sarcomas of the trunk wall (STS-TW): a study of 343 patients from the French Sarcoma Group (FSG) database. Ann Oncol 20(6): 1127-1135.

Schemper M, Smith TL (1996) A note on quantifying follow-up in studies of failure time. Control Clin Trials 17(4): 343-346.

Singer S, Demetri GD, Baldini EH, Fletcher CD (2000) Management of soft-tissue sarcomas: an overview and update. The lancet oncology 1 : 75-85.

Stojadinovic A, Leung DH, Allen P, Lewis JJ, Jaques DP, Brennan MF (2002a) Primary adult soft tissue sarcoma: time-dependent influence of prognostic variables. J Clin Oncol 20(21): 4344-4352.
Stojadinovic A, Leung DH, Hoos A, Jaques DP, Lewis JJ, Brennan MF (2002b) Analysis of the prognostic significance of microscopic margins in 2,084 localized primary adult soft tissue sarcomas. Ann Surg 235(3): 424-434.

Trovik CS, Bauer HC, Alvegard TA, Anderson H, Blomqvist C, Berlin O, Gustafson P, Saeter G, Walloe A (2000a) Surgical margins, local recurrence and metastasis in soft tissue sarcomas: 559 surgically-treated patients from the Scandinavian Sarcoma Group Register. Eur J Cancer 36(6): 710-716.

Trovik CS, Bauer HC, Berlin O, Tukiainen E, Erlanson M, Gustafson P, Klepp R, Saeter G, Wahlstrom O (2001) Local recurrence of deep-seated, high-grade, soft tissue sarcoma: 459 patients from the Scandinavian Sarcoma Group Register. Acta Orthop Scand 72(2): 160-166.

Trovik CS, Gustafson P, Bauer HC, Saeter G, Klepp R, Berlin O, Erlanson M, Wahlstrom O, Raabe N (2000b) Consequences of local recurrence of soft tissue sarcoma: 205 patients from the Scandinavian Sarcoma Group Register. Acta Orthop Scand 71(5): 488-495.

Ueda T, Yoshikawa H, Mori S, Araki N, Myoui A, Kuratsu S, Uchida A (1997) Influence of local recurrence on the prognosis of soft-tissue sarcomas. $J$ Bone Joint Surg Br 79(4): 553-557.

Weitz J, Antonescu CR, Brennan MF (2003) Localized extremity soft tissue sarcoma: improved knowledge with unchanged survival over time. J Clin Oncol 21(14): 2719-2725.

Zagars GK, Ballo MT, Pisters PW, Pollock RE, Patel SR, Benjamin RS, Evans HL (2003) Prognostic factors for patients with localized soft-tissue sarcoma treated with conservation surgery and radiation therapy: an analysis of 1225 patients. Cancer 97(10): 2530-2543.

This work is published under the standard license to publish agreement. After 12 months the work will become freely available and the license terms will switch to a Creative Commons AttributionNonCommercial-Share Alike 3.0 Unported License. 